\title{
SNAI2 is induced by transforming growth factor- $\beta 1$, but is not essential for epithelial-mesenchymal transition in human keratinocyte HaCaT cells
}

\author{
YUKI MIYAKE $^{1,2}$, YOSHIYUKI NAGAOKA ${ }^{1}$, KAZUHIKO OKAMURA $^{3}$, YUKIMASA TAKEISHI ${ }^{1}$, \\ SACHIO TAMAOKI $^{2}$ and MITSUTOKI HATTA ${ }^{1,4}$
}

Departments of ${ }^{1}$ Physiological Science and Molecular Biology, ${ }^{2}$ Oral Growth and Development and ${ }^{3}$ Morphological Biology;
${ }^{4}$ Oral Medicine Research Center, Fukuoka Dental College, Fukuoka 814-0193, Japan

Received March 4, 2021; Accepted July 6, 2021

DOI: $10.3892 / \mathrm{etm} .2021 .10558$

\begin{abstract}
Epithelial-mesenchymal transition (EMT) is a cellular process in which epithelial cells lose their epithelial traits and shift to the mesenchymal phenotype, and is associated with various biological events, such as embryogenesis, wound healing and cancer progression. The transcriptional program that promotes phenotype switching is dynamically controlled by transcription factors during EMT, including Snail (SNAI1), twist family bHLH transcription factor (TWIST) and zinc finger E-box binding homeobox 1 (ZEB1). The present study aimed to investigate the molecular mechanisms underlying EMT in squamous epithelial cells. Western blot analysis and immunocytochemical staining identified Slug (SNAI2) as a transcription factor that is induced during transforming growth factor (TGF)- $\beta 1$-mediated EMT in the human keratinocyte cell line HaCaT. The effect of SNAI2 overexpression and knockdown on the phenotypic characteristics of $\mathrm{HaCaT}$ cells was evaluated. Filamentous actin staining and western blot analysis revealed that the overexpression of SNAI2 did not induce the observed EMT-related phenotypic changes. In addition, SNAI2 knockdown demonstrated almost no impact on the EMT phenotypes induced by TGF- $\beta 1$. Notably, DNA microarray analysis followed by comprehensive bioinformatics analysis revealed that the differentially expressed genes upregulated by TGF- $\beta 1$ were significantly enriched in cell adhesion and extracellular matrix binding, whereas the genes downregulated in response to TGF- $\beta 1$ were significantly enriched in the cell cycle. No enriched gene ontology term and biological pathways were identified in the differentially expressed gene sets of SNAI2-overexpressing cells. In addition, the candidates
\end{abstract}

Correspondence to: Professor Mitsutoki Hatta, Department of Physiological Science and Molecular Biology, Fukuoka Dental College, 2-15-1 Tamura, Fukuoka 814-0193, Japan

E-mail: hatta@college.fdcnet.ac.jp

Key words: transforming growth factor- $\beta 1$, epithelial-mesenchymal transition, keratinocyte, Slug (SNAI2), enrichment analysis for master transcription factors regulating the TGF- $\beta 1$-induced EMT were identified using transcription factor enrichment analysis. In conclusion, the results of study demonstrated that SNAI2 does not play an essential role in the EMT of HaCaT cells and identified candidate transcription factors that may be involved in EMT-related gene expression induced by TGF- $\beta 1$. These findings may enhance the understanding of molecular events in EMT and contribute to the development of a novel therapeutic approach against EMT in cancers and wound healing.

\section{Introduction}

Under certain circumstances, such as embryogenesis, organ development, tissue regeneration, and tumor progression and metastasis, epithelial cells are able to induce a biological process known as epithelial-mesenchymal transition (EMT) $(1,2)$. During EMT, epithelial cells lose their epithelial characteristics (cell-cell contact and epithelial polarity) and transform into a mesenchymal phenotype (higher motility and cell-extracellular matrix interactions) $(1,2)$. EMT has been classified into three types based on the biological events during which it occurs $(2,3)$. Type 1 EMT is associated with developmental processes, such as gastrulation and neural crest cell migration. This embryological EMT occurs in the orofacial region during palate formation $(2,3)$. Type 2 EMT is associated with wound healing. Under certain conditions, such as prolonged inflammation, type 2 EMT can lead to fibrosis of damaged tissues mediated by enhanced cytokine production, such as TGF- $\beta 1$ and TNF $\alpha(2,3)$. Type 3 EMT is associated with tumor progression and metastasis. Cancer cells acquire an invasiveness and metastatic potential through induction of the EMT process in the tumor microenvironment $(2,3)$.

It has been reported that a subset of transcription factors, including members of the snail family transcriptional repressor (SNAI), twist family bHLH transcription factor (TWIST), and zinc finger E-box binding homeobox (ZEB) families, trigger changes in dynamic gene expression that include the suppression of epithelial phenotypic genes, such as E-cadherin, claudin and cytokeratin as well as the induction of mesenchymal genes, such as N-cadherin and fibronectin $(4,5)$. SNAI2 (Slug) 
is an EMT transcription factor of the SNAI family and is known to be involved in type 2 EMT during cutaneous wound healing (6-8). Epithelial cells at the edge of the wound undergo partial EMT in which the cells attain a hybrid epithelial/mesenchymal phenotype enabling them to move to the damaged area and re-constitute healthy epithelial tissue (9). It is thought that the partial EMT of squamous epithelial cells is mediated by SNAI2, which in turn is mediated by the epidermal growth factor receptor $(6,7)$. In our previous study, elevated SNAI2 mRNA levels were observed during TGF- $\beta 1$-induced EMT in the human keratinocyte cell line HaCaT (10). These findings suggested that SNAI2 triggers the EMT transcription program and plays a critical role in managing the dynamic behavior of squamous epithelial cells.

The present study aimed to investigate the molecular mechanisms underlying EMT in squamous epithelial cells. Thus, the role of SNAI2 in a TGF- $\beta 1$-induced EMT model in $\mathrm{HaCaT}$ cells was examined. The findings of the present study may advance the understanding of the molecular mechanisms underlying EMT, and contribute to the identification of novel therapeutic targets for diseases and pathological conditions, such as cancers and wound healing.

\section{Materials and methods}

Cell culture. HaCaT cells (a gift from Dr Tetsuro Ikebe, Fukuoka Dental College, Japan), NIH3T3 cells (a gift from Dr Tsuyako Ohkubo, Fukuoka Nursing College, Japan) and GP2-293 cells (cat. no. 631458; Takara Bio, Inc.) were cultured in a medium consisting of DMEM (cat. no. 08458-45; Nacalai Tesque, Inc.) supplemented with 10\% FBS (cat. no. S1820-500; BioWest), $50 \mathrm{U} / \mathrm{ml}$ penicillin and $50 \mu \mathrm{g} / \mathrm{ml}$ streptomycin. The cells were maintained by subculturing twice a week at $37^{\circ} \mathrm{C}$ with $5 \% \mathrm{CO}_{2}$. Short tandem repeat analysis was performed using a GenePrint 24 System (cat. no. B1870; Promega Corporation) in order to authenticate the HaCaT cell line. To induce EMT in HaCaT cells, $20 \mathrm{ng} / \mathrm{ml}$ recombinant TGF- $\beta 1$ (cat. no. 5231; Cell Signaling Technology, Inc.) was added to the culture medium and the cells were cultured for $48 \mathrm{~h}$ at $37^{\circ} \mathrm{C}$ with $5 \% \mathrm{CO}_{2}$ as previously described (10). The morphology of the cells was observed with a phase contrast microscope (Nikon Corporation; magnification, x100).

Plasmid construction and generation of SNAI2 overexpressing cells. The pMXs-TY1 plasmid was generated by inserting DNA fragments corresponding to TY1-tag into the BamH1-EcoRI site of the pMXs-puro retrovirus vector (Cell Bio, Inc.). PCR-amplification of human SNAI2 cDNA was carried out using PrimeSTAR ${ }^{\circledR}$ Max DNA polymerase (cat. no. R045A; Takara Bio, Inc.) with the following primers (5'-CGCGGATCCCCATGCCGCGCTCCTTCC TGGTCAAG-3' and 5'-CGCGGATCCTCAGTGTGCTAC ACAGCAGCCAGA-3'). The thermocycling conditions were as follows: $94^{\circ} \mathrm{C}$ for $1 \mathrm{~min}$; followed by 40 cycles at $98^{\circ} \mathrm{C}$ for $10 \mathrm{sec}, 55^{\circ} \mathrm{C}$ for $15 \mathrm{sec}$ and $72^{\circ} \mathrm{C}$ for $30 \mathrm{sec}$; followed by final extension at $72^{\circ} \mathrm{C}$ for $2 \mathrm{~min}$. cDNA was subcloned into the pMXs-TY1 (pMXs-TY1-SNAI2). Lipofectamine $2000^{\circledR}$ (Invitrogen; Thermo Fisher Scientific, Inc.) was used to co-transfect $1 \mu \mathrm{g}$ of the pMXs-TY1 or pMXs-TY1-SNAI2 plasmid and $1 \mu \mathrm{g}$ of the p10A1 vector (1:1) into the GP2-293 cells $\left(1 \times 10^{6}\right.$ cells; cat. no. 631458; Takara Bio, Inc.), which is a HEK 293-based retroviral packaging cell line stably expressing the MoMuL V Gag and Pol proteins. At $48 \mathrm{~h}$ post-transfection, the virus-containing supernatants were collected and added to the HaCaT-cell growth medium, followed by the addition of $7.5 \mu \mathrm{g} / \mathrm{ml}$ polybrene. HaCaT cells $\left(5 \times 10^{5}\right.$ cells) were cultured for $24 \mathrm{~h}$ in the virus-containing medium, which was then replaced with fresh growth medium. A total of $48 \mathrm{~h}$ after virus transduction, the cells were cultured in the $\mathrm{HaCaT}$ cell growth medium containing $2 \mu \mathrm{g} / \mathrm{ml}$ puromycin (Sigma-Aldrich; Merck KGaA) to enable the selection of stably transduced cells.

Establishment of SNAI2-knockdown cells. Oligonucleotides for generating short hairpin (sh)RNA-targeting human SNAI2 (shSNAI2; 5'-GATCCGGCATTTGCAGAC AGGTCAAATTTCAAGAGAATTTGACCTGTCTGCAAA TGCCTTTTTTGCTAGCG-3') and the non-targeting control (shNC; 5'-GATCCGTGCGTTGCTAGTACCAACTTCAAG AGATTTTTTACGCGTG-3') were synthesized. Fragments of annealed oligonucleotides were cloned into a pSIREN-RetroQ retroviral shRNA expression vector (a gift from Dr Kazuya Yamagata, Kumamoto University, Japan). Retrovirus-mediated gene transfer was used to establish stable cells expressing shRNA. Briefly, $1 \mu \mathrm{g}$ of the shRNA expression plasmid (pSIREN-RetroQ-shSNAI2 or pSIREN-RetroQ-shNC) and $1 \mu \mathrm{g}$ of the p10A1 vector (1:1) were transfected into the GP2-293 cells $\left(1 \times 10^{6}\right.$ cells; Takara Bio, Inc.) using Lipofectamine $2000^{\circledR}$ (Invitrogen; Thermo Fisher Scientific, Inc.) at $37^{\circ} \mathrm{C}$ for $48 \mathrm{~h}$, after which the virus-containing supernatants were collected and immediately added to the HaCaT-cell growth medium, followed by the addition of $7.5 \mu \mathrm{g} / \mathrm{ml}$ polybrene. The retroviral transduction of $\mathrm{HaCaT}$ cells was performed as described previously.

Reverse transcription-quantitative $(R T-q) P C R$. Extraction of total RNA from HaCaT cells was performed using a NucleoSpin RNA kit (cat. no. 740955.50; Machrey-Nagel, $\mathrm{GmBH}$ ) and reverse-transcription into cDNA was performed using a PrimeScript ${ }^{\mathrm{TM}}$ RT Master Mix (cat. no. RR036A; Takara Bio, Inc.) according to the manufacturer's instructions. Fluorescence dye-based qPCR was performed on a CFX96 Real-time System (Bio-Rad Laboratories, Inc.) using TB Green Premix Ex Taq II (cat. no. RR820A; Takara Bio, Inc.). The primers used were as follows SNAI2 forward, 5'-CGA ACTGGACACACATACAGTG-3' and reverse, 5'-CTGAGG ATCTCTGGTTGTGGT-3'; and GAPDH, forward 5'-GGA GCGAGATCCCTCCAAAAT-3' and reverse, 5'-GGCTGT TGTCATACTTCTCATGG-3'. The thermocycling conditions were as follows: $95^{\circ} \mathrm{C}$ for $30 \mathrm{sec}$; followed by 40 cycles at $95^{\circ} \mathrm{C}$ for $5 \mathrm{sec}$ and $60^{\circ} \mathrm{C}$ for $30 \mathrm{sec}$. mRNA expression levels were normalized to the levels of GAPDH and the $2^{-\Delta \Delta \mathrm{Cq}}$ method was used for relative quantification (CFX Manager version 3.1; Bio-Rad Laboratories, Inc.) (11).

Western blotting. Total protein was isolated from the cells using a lysis buffer [50 mM Tris- $\mathrm{HCl}(\mathrm{pH} 8.0), 300 \mathrm{mM}$ $\mathrm{NaCl}, 1 \% \mathrm{SDS}]$ and quantified using a BCA assay. Total protein extracts $(10-20 \mu \mathrm{g})$ were resolved by electrophoresis in $4-20 \%$ polyacrylamide-SDS gels and transferred onto 
PVDF membranes. The membranes were blocked with $0.1 \%$ Tween-TBS (TBST) containing 4\% (w/v) ECL Prime Blocking Agent (cat. no. RPN418; Cytiva) or by using an EzBlock Chemi (cat. no. AE-1475; ATTO), according to the manufacturer's instructions. The membranes were then incubated with primary antibodies for specific proteins for $1.5 \mathrm{~h}$ at $21-25^{\circ} \mathrm{C}$ or overnight at $4^{\circ} \mathrm{C}$. After washing with TBST for 3 times at $10 \mathrm{~min}$ each, the membranes were incubated with horseradish peroxidase (HRP)-conjugated secondary antibodies against mouse $\mathrm{IgG}$ or rabbit $\mathrm{IgG}$ for $1 \mathrm{~h}$ at $21-25^{\circ} \mathrm{C}$. Chemiluminescence reactions were detected using a LAS-4000 imaging system (Cytiva) and the intensity of each band was quantified using ImageJ v.1.53a (National Institutes of Health). Expression levels were normalized to the levels of $\beta$-actin. The primary antibodies used in the present study were as follows: Anti-Slug (SNAI2; 1:2,500; cat. no. 9585; Cell Signaling Technology Inc.), anti-E-cadherin (1:2,500; cat. no. 3195; Cell Signaling Technology Inc.), anti-N-cadherin (1:2,500; cat. no. 13116; Cell Signaling Technology, Inc.), anti-Claudin-1 (1:2,500; cat. no. 13255, Cell Signaling Technology, Inc.), anti-Snail (1:2,500; cat. no. 3879; Cell Signaling Technology, Inc.), anti-Twist (1:2,500; cat. no. ab50887; Abcam), anti-Cytokeratin 13 (1:2,500; cat. no. ab92551; Abcam), anti-Cytokeratin 15 (1:2,500; cat. no. ab52816; Abcam), anti-Fibronectin/NF1 (1:2,500; cat. no. 26836; Cell Signaling Technology Inc.), anti-TCF8/ZEB1 (1:2,500; cat. no. 3396; Cell Signaling Technology Inc.), anti- $\beta$-Actin (1:5,000; cat.no. sc-69879; Santa Cruz Biotechnology, Inc.), anti-mouse IgG HRP-conjugated antibody (1:2,500; cat. no. 7076; Cell Signaling Technology, Inc.) and anti-rabbit IgG HRP-conjugated antibody $(1: 2,500$; cat. no. 7074; Cell Signaling Technology, Inc.).

Immunocytochemical staining. Immunocytochemical staining was performed as in our previous study (10). Briefly, HaCaT cells were plated on Nunc Lab-Tek Chamber Slides (cat. no. 177429; Thermo Fisher Scientific Inc.) and cultured with or without TGF- $\beta 1$ for $48 \mathrm{~h}$ at $37^{\circ} \mathrm{C}$ with $5 \% \mathrm{CO}_{2}$. The cells were fixed with $4 \%$ paraformaldehyde in PBS for $20 \mathrm{~min}$ at $21-25^{\circ} \mathrm{C}$, permeabilized with $0.1 \%$ Triton X-100 in PBS for $3 \mathrm{~min}$, and then washed 3 times with PBS. Staining of filamentous actin (F-actin) was achieved by blocking with $1 \% \mathrm{BSA}$ in PBS for $30 \mathrm{~min}$ at $21-25^{\circ} \mathrm{C}$, followed by probing with Alexa Fluor 488 Phalloidin (1:50; cat. no. A-12379; Thermo Fisher Scientific, Inc.) for $1 \mathrm{~h}$ at $21-25^{\circ} \mathrm{C}$. Staining of SNAI2 was carried out by probing first with an anti-Slug (1:100; cat. no. 9585; Cell Signaling Technology, Inc.) for $2 \mathrm{~h}$ at $21-25^{\circ} \mathrm{C}$ and then anti-rabbit IgG antibody conjugated with Alexa Fluor 488 (1:800; cat. no. A-11008; Thermo Fisher Scientific, Inc.) for $1.5 \mathrm{~h}$ at $21-25^{\circ} \mathrm{C}$. After staining, DAPI Fluoromount-G ${ }^{\circledR}$ (cat. no. 0100-20; SouthernBiotech) was used to visualize nuclei and preserve fluorescence. Finally, fluorescent images were captured by a fluorescence microscope (BZ-9000; Keyence Corporation; magnification, x200).

DNA microarray. Total RNA of the non-treated HaCaT, TGF- $\beta 1$-treated $(20 \mathrm{ng} / \mathrm{ml}$ TGF- $\beta 1$; treated for $48 \mathrm{~h}$ at $37^{\circ} \mathrm{C}$ ), TY1 tag-overexpressing and TY1-tagged SNAI2-overexpressing cells was extracted using a NucleoSpin RNA kit (Machrey-Nagel, GmBH \& Co.). The cRNA was amplified and labeled using a Low-Input QuickAmp Labeling kit (Agilent Technologies, Inc.) and hybridized to a SurePrint G3 Human Gene Expression Microarray 8x60K v3 (Agilent Technologies, Inc.). The procedure was carried out as previously described (12-14). All hybridized microarray slides were scanned by an Agilent scanner, after which relative hybridization intensities and background hybridization values were calculated using Agilent Feature Extraction Software (9.5.1.1). The raw signal intensities and flags for each probe were calculated and normalized by a quantile algorithm. Identification of differentially expressed genes was carried out by calculating Z-scores and non-log scaled fold-change ratios. Criteria for upregulated genes were $Z$-score $>2.0$ and ratio $>2.0$, whereas those for downregulated genes were Z-score <-2.0 and ratio $<0.5$. The DNA microarray datasets were deposited in the Gene Expression Omnibus (GEO) database (https://www.ncbi. nlm.nih.gov/geo/) at the National Center for Biotechnology Information (NCBI) with the accession numbers GSE166199 and GSE166200.

Heatmap and gene ontology (GO) and pathway enrichment analyses. A heatmap was generated as previously described (15) and genes were sorted using a hierarchical clustering technique, with the distance from the median of each row indicated by color. The ToppGene Suite (https//toppgene.cchmc.org) (16) was used to perform both the GO pathway analysis of biological processes, cell components, and molecular functions, and the Kyoto Encyclopedia of Genes and Genomes (KEGG) pathway analysis of genes differentially expressed between non-treated and TGF- $\beta$-treated HaCaT cells, or between TY1-tag and TY1-tagged SNAI2-overexpressing cells.

Transcription factor binding site enrichment analysis. ChIP-X Enrichment Analysis 3 (https://amp.pharm.mssm. edu/ChEA3) (17) was used to identify the transcription factors responsible for TGF- $\beta$-mediated EMT in HaCaT cells.

Statistical analysis. Data are expressed as the mean \pm standard error of the mean (SEM) of 3-6 biological replicates for each group. Statistical analysis was performed using KaleidaGraph 4.5 (Hulinks, Inc.) Statistical differences were calculated using an unpaired Student's t-test for two groups or one-way analysis of variance (ANOVA) followed by the post hoc Bonferroni's test for multiple comparisons. $\mathrm{P}<0.05$ was considered to indicate a statistically significant difference.

\section{Results}

Increased expression of SNAI2 in HaCaT cells during TGF- $\beta 1$-mediated EMT. To elucidate the molecular mechanisms of the EMT transcription program, an in vitro model of TGF- $\beta 1$-induced EMT in HaCaT cells (10) was used. Firstly, the TGF- $\beta 1$-induced phenotypic changes in HaCaT cells were determined. The change in morphology from a paving stone-like appearance to a spindle-shape was observed under a phase contrast microscope (Fig. 1A). In addition, F-actin staining indicated that well-aligned actin stress fibers were formed as a result of TGF- $\beta 1$ treatment (Fig. 1B). Western blot analysis was carried out to examine the degree to which mesenchymal markers and epithelial markers were expressed. The mesenchymal marker fibronectin was substantially increased, 
A

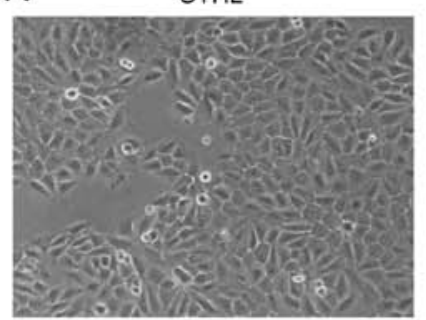

TGF- $\beta 1$

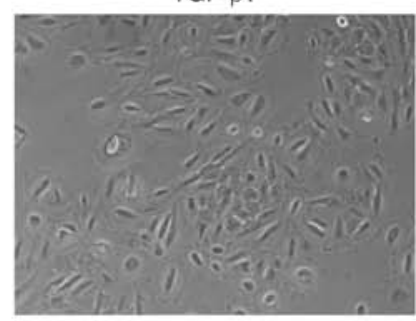

B

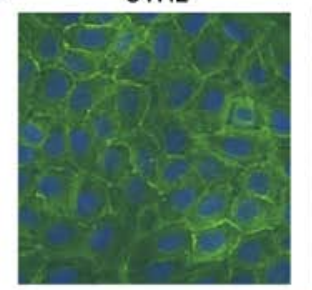

Phalloidin DAP

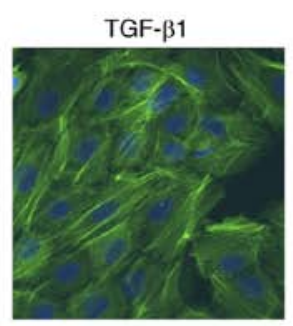

C
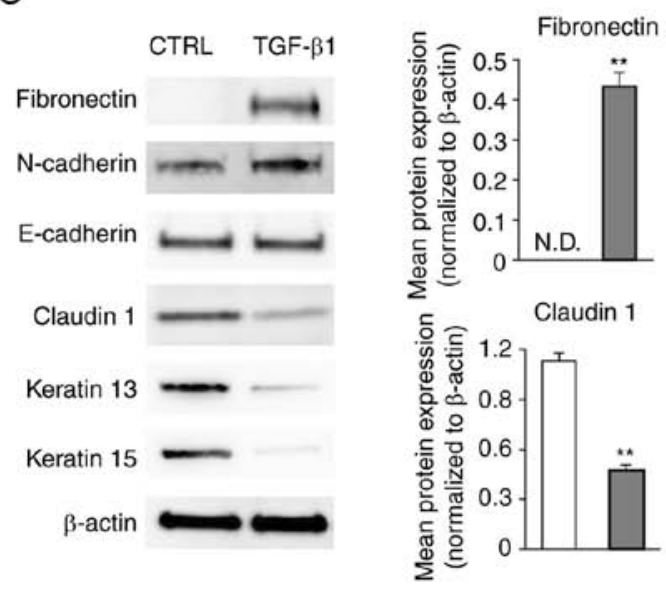

口CTRL $\square$ TGF- $\beta 1$
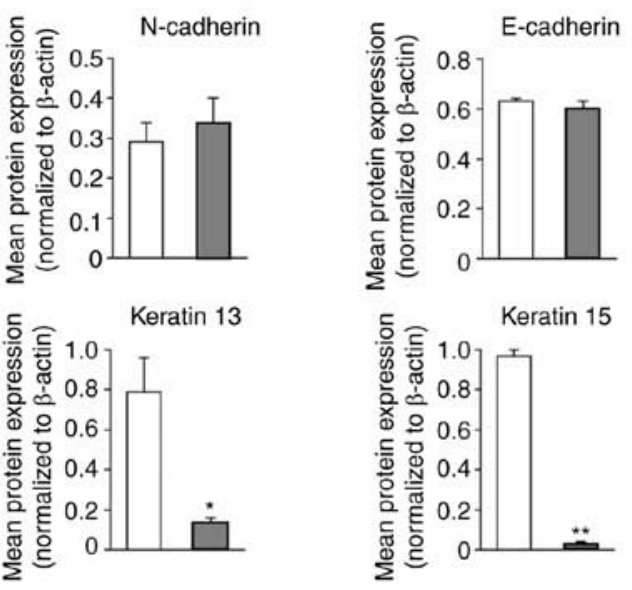

E
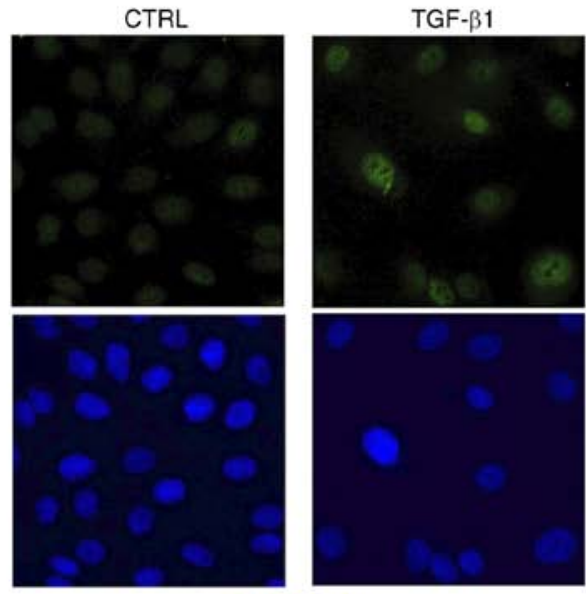

SNAI2 DAPI

Figure 1. SNAI2 is increased in HaCaT cells during TGF- $\beta 1$-mediated EMT. (A) Changes in the cell morphology of HaCaT cells were observed under a phase contrast microscope. Representative images are shown (magnification, x100). (B) The distribution of F-actin was visualized by staining with fluorescence-conjugated phalloidin and the nuclei were labeled with DAPI. Representative images are shown (magnification, x200). (C) Changes in the mesenchymal markers (fibronectin and N-cadherin) and the epithelial markers (E-cadherin, claudin1, keratin 13 and keratin 15) were observed by western blotting. Representative images are shown. Protein levels were quantified by calculating the band intensity and normalized to the levels of $\beta$-actin. Data are shown as the mean (bar) and SEM (whisker). ${ }^{*} \mathrm{P}<0.05,{ }^{* *} \mathrm{P}<0.01$. (D) Expression levels of EMT transcription factors (SNAI1, SNAI2, TWIST1/2 and ZEB1) were analyzed by western blotting. NIH3T3 cells were used for positive controls of each antibodies. Representative images are shown. SNAI2 protein levels were quantified by calculating the band intensity and normalized to the levels of $\beta$-actin. Data are shown as the mean (bar) and SEM (whisker). ${ }^{* *} \mathrm{P}<0.01$. (E) SNAI2 expression was examined by immunofluorescence staining and nuclei were visualized with DAPI staining. The representative images shown here were obtained using a conventional fluorescence microscope (magnification, x200). EMT, epithelial-mesenchymal transition; SNAI2, Slug; ND, not detectable; TGF- $\beta 1$, transforming growth factor- $\beta 1$; CTRL, control (non-treated); SNAI1, snail family transcriptional repressor 1; ZEB1, zinc finger E-box binding homeobox 1 ; TWIST $1 / 2$, twist family bHLH transcription factor $1 / 2$.

whereas the epithelial markers claudin 1 , keratin 13 and keratin 15 were decreased in EMT-induced HaCaT cells (18) $(\mathrm{P}<0.05$ or $\mathrm{P}<0.01$; Fig. $1 \mathrm{C})$. However, no significant changes in the protein levels of $\mathrm{N}$-cadherin and $\mathrm{E}$-cadherin were found (Fig. 1C). Our previous study revealed that the mRNA levels of the EMT transcription factors SNAI2 and TWIST1 were increased by TGF- $\beta 1$ in $\mathrm{HaCaT}$ cells (10). Hence, the protein levels of these factors were evaluated by western blotting in the present study. SNAI2, but not SNAI1, TWIST, or ZEB1, was expressed in $\mathrm{HaCaT}$ cells, and its protein levels were 

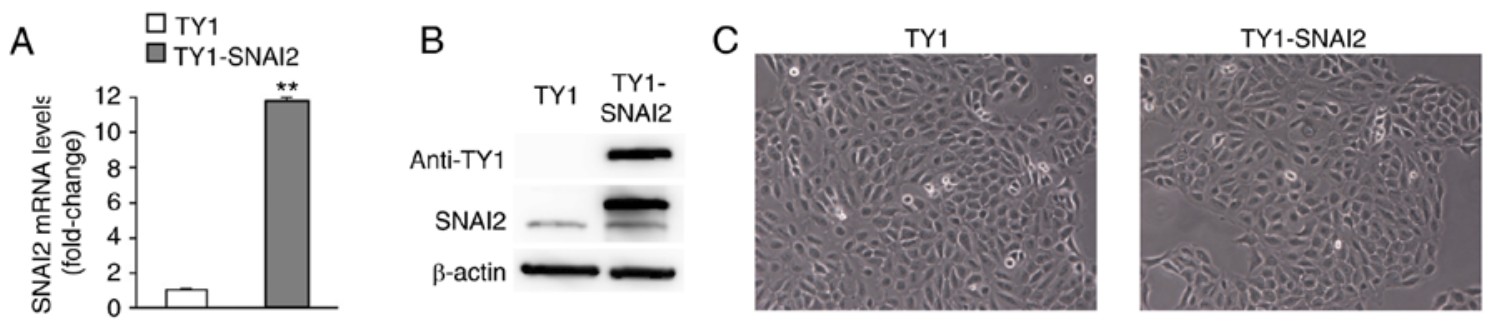

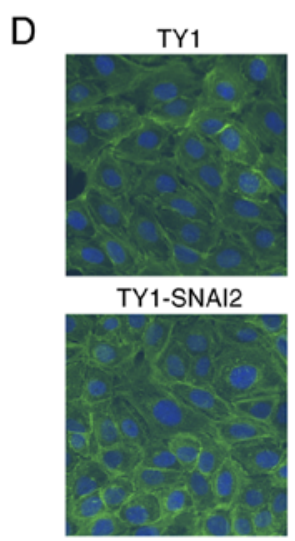

Phalloidin DAPI
$\mathrm{E}$

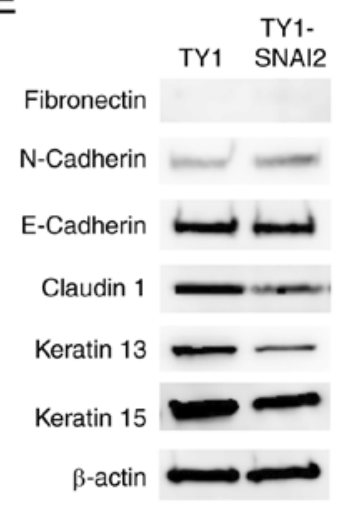

Figure 2. SNAI2 overexpression is unable to induce EMT features in HaCaT cells. (A) SNAI2 mRNA levels in HaCaT cells expressing TY1-tag (as a control) or TY1-SNAI2 were examined by RT-qPCR. mRNA levels were normalized to GAPDH levels and calculated relative to that of control cells. Data are the mean (bar) plus SEM (whisker). ${ }^{* *} \mathrm{P}<0.01$. (B) SNAI2 protein levels were evaluated using western blotting. Representative images are shown. (C) Cell morphology of $\mathrm{HaCaT}$ cells was examined under a phase contrast microscope. Representative images are shown (magnification, x100). (D) Distribution of F-actin was visualized by staining with fluorescence-conjugated phalloidin and the nuclei were labeled with DAPI. Representative images are shown (magnification, x200). (E) Mesenchymal markers (fibronectin and N-cadherin) and the epithelial markers (E-cadherin, claudin1, keratin 13 and keratin 15) were analyzed by western blotting. Representative images are shown. The protein levels were quantified by calculating the band intensity and normalized to the levels of $\beta$-actin. Data are shown as the mean (bar) and SEM (whisker). ${ }^{* *}$ P<0.01. N.D., not detectable; RT-q; reverse transcription-quantitative; SNAI2, Slug; TY-1, TY1-tag (TY1-tag expressing).

significantly increased by TGF- $\beta 1$ treatment $(\mathrm{P}<0.01$; Fig. $1 \mathrm{D})$. The lysate of mouse embryonic fibroblast-derived NIH3T3 cells was used as a positive control for the protein expression of EMT transcription factors (19). In addition, immunocytochemical staining demonstrated that SNAI2 was increased in the EMT-induced HaCaT cells compared with the non-treated cells (Fig. 1E). These data suggested that TGF- $\beta 1$ induced SNAI2 expression and partially promoted the EMT-related alterations of epithelial and mesenchymal markers in $\mathrm{HaCaT}$ cells.

Overexpression or knockdown of SNAI2 has less impact on the phenotypic characteristics of $\mathrm{HaCaT}$ cells. To investigate whether SNAI2 serves a critical role in the TGF- $\beta 1$-induced EMT of HaCaT cells, TY1-tagged SNAI2-overexpressing HaCaT cells (TY1-SNAI2) were generated and the enhanced expression of SNAI2 was confirmed by RT-qPCR and western blotting $(\mathrm{P}<0.01$; Fig. $2 \mathrm{~A}$ and $\mathrm{B})$. There was no significant difference in cell morphology, including cell shape and cytoskeletal architecture, between SNAI2-overexpressing cells and their corresponding control cells (TY1) (Fig. 2C and D). Subsequently, EMT-marker expression was evaluated by western blotting. SNAI2-overexpression did not induce the expression of fibronectin and no marked changes in the protein levels of $\mathrm{N}$-cadherin, E-cadherin, and keratin 15 were observed (Fig. 2E). However, it was found that both claudin 1, which is involved in epithelial cell-cell adhesion, and keratin 13 cytoskeletal protein were decreased in the SNAI2 overexpressing cells compared with the control cells $(20,21)$ $(\mathrm{P}<0.01$; Fig. 2E).

Next, SNAI2-knockdown HaCaT cells (shSNAI2) were created by using the shRNA expression system. The level of SNAI2 mRNA was reduced to $50 \%$ compared with the shNC $(\mathrm{P}<0.01$; Fig. 3A). Cell morphology was not significantly changed in the SNAI2-knockdown cells under the control condition (Fig. 3B). Both shNC and shSNAI2 cells were treated with TGF- $\beta 1$ in order to investigate the effect of SNAI2 knockdown on the EMT phenotype. The same levels of EMT-related morphological change were induced by TGF- $\beta 1$ in both SNAI2-knockdown and control cells (Fig. 3B), and F-actin staining demonstrated no significant difference between the two types of cells (Fig. 3C). Western blotting was performed to examine the expression of SNAI2 and other EMT markers. As shown in Fig. 3D, SNAI2 protein level was effectively decreased and knockdown had a large impact on the TGF- $\beta 1$-induced expression of SNAI2. The protein levels of the epithelial markers claudin 1, keratin 13 and keratin 15 were increased in SNAI2-knockdown cells compared with control cells. Furthermore, the protein levels of claudin 1 and keratin 15 , but not keratin 13, were downregulated by TGF- $\beta 1$ $(\mathrm{P}<0.05$ or $\mathrm{P}<0.01$; Fig. 3D). The induction of fibronectin in response to TGF- $\beta 1$ and the expression levels of $\mathrm{N}$-cadherin and E-cadherin were not significantly changed in either group of cells (Fig. 3D). These data suggested that SNAI2 affected 
B

A

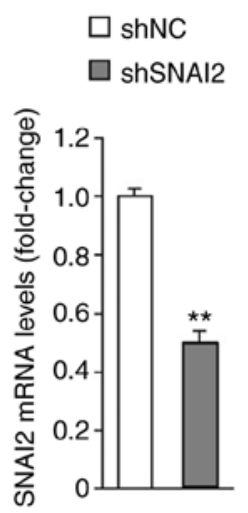

B
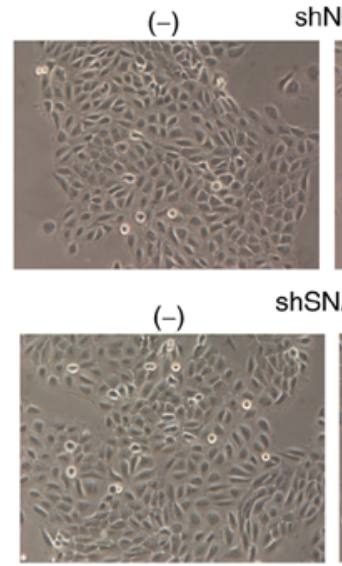

TGF- $\beta 1$

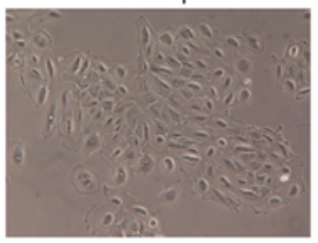

SNAI2 TGF- $\beta 1$

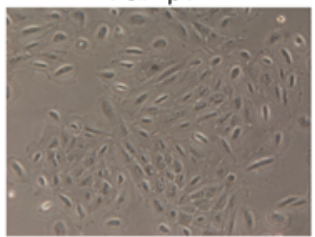

C

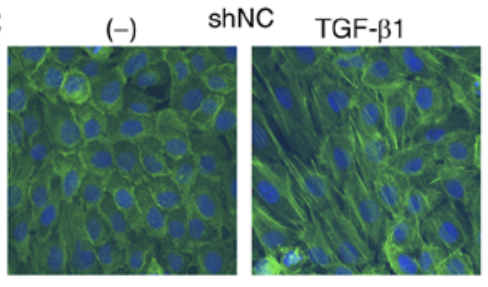

(-) ShSNAI2 TGF- $\beta 1$

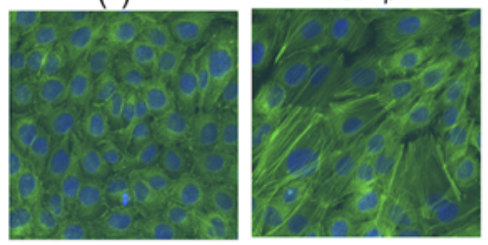

Phalloidin DAPI
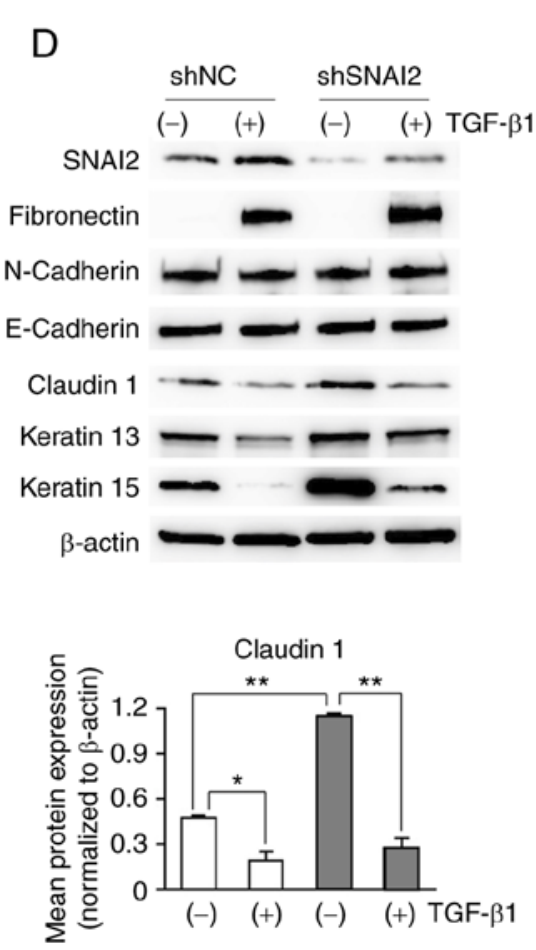
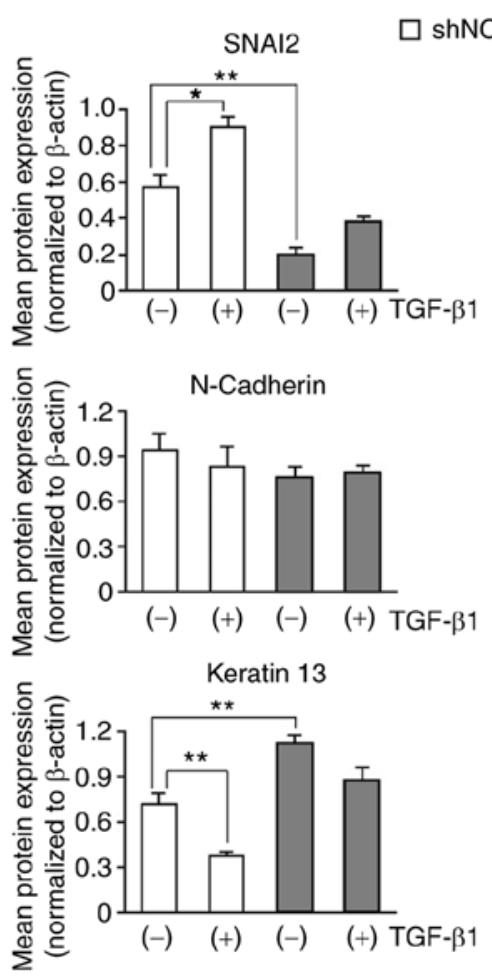
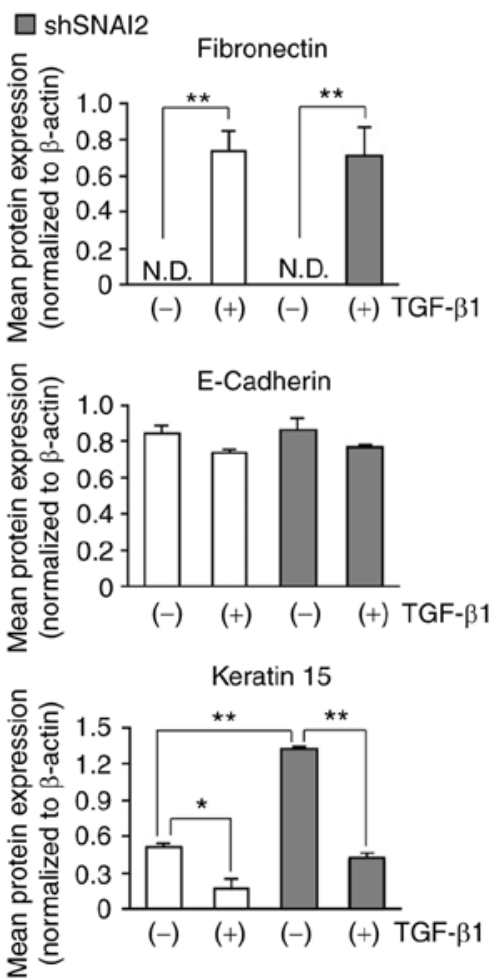

Figure 3. Knockdown of SNAI2 does not affect the TGF- $\beta 1$-induced EMT phenotype of HaCaT cells, compared with the non-targeting control. (A) SNAI2 mRNA levels in shNC (as a control) and shSNAI2 HaCaT cells were evaluated using RT-qPCR. The fold change in mRNA levels was normalized to the levels of GAPDH and calculated relative to that of control cells. Data are the mean (bar) plus SEM (whisker) ${ }^{* *} \mathrm{P}<0.01$. (B) Cell morphology of shNC and shSNAI2 $\mathrm{HaCaT}$ cells with or without TGF- $\beta 1$ treatment were examined using a phase contrast microscope. Representative images are shown (magnification, x100). (C) Organization of F-actin was examined by staining with fluorescence-conjugated phalloidin and the nuclei were labeled with DAPI. Representative images are shown (magnification, x200). (D) SNAI2, the mesenchymal markers (fibronectin and N-cadherin) and the epithelial markers (E-cadherin, claudin1, keratin 13 and keratin 15) were analyzed by western blotting. Representative images are shown. The protein levels were quantified by calculating the band intensity and normalized to the levels of $\beta$-actin. Data are shown as the mean (bar) and SEM (whisker). ${ }^{*} \mathrm{P}<0.05,{ }^{* * *} \mathrm{P}<0.01$. N.D., not detectable; sh, short hairpin; $\mathrm{NC}$, negative control (non-targeting); RT-q, reverse transcription-quantitative; TGF- $\beta 1$, transforming growth factor- $\beta 1$.

a subset of the epithelial markers but was unable to induce the EMT-related markers in HaCaT cells. In addition, SNAI2 knockdown had less of an impact on the TGF- $\beta 1$-induced EMT.

Identification of differentially expressed genes. DNA microarray was performed to elucidate the molecular mechanism underlying the difference between TGF- $\beta 1$-induced EMT and the directed overexpression of SNAI2. The hierarchical clustering analysis demonstrated significant changes in the gene expression profile of TGF- $\beta 1$-treated HaCaT cells compared with control cells (Fig. 4A). A total of 507 downregulated and 544 upregulated genes (a 2-fold change) were observed on TGF- $\beta 1$ treatment (Fig. 4A). Hierarchical clustering also revealed that induced expression of SNAI2 downregulated 133 genes and upregulated 90 genes in HaCaT cells (Fig. 4B). In addition, it was investigated whether SNAI2-induced transcriptomic changes overlapped with that of TGF- $\beta 1$-induced EMT in HaCaT cells. As demonstrated in Fig. 4C, four genes; RNF157-AS1 (RNF157 antisense RNA 1), SCGB1A1 (secretoglobin family $1 \mathrm{~A}$ member 1 ), S100P (S100 calcium binding protein P) and OLFM4 (olfactomedin 4) were identified as 
A

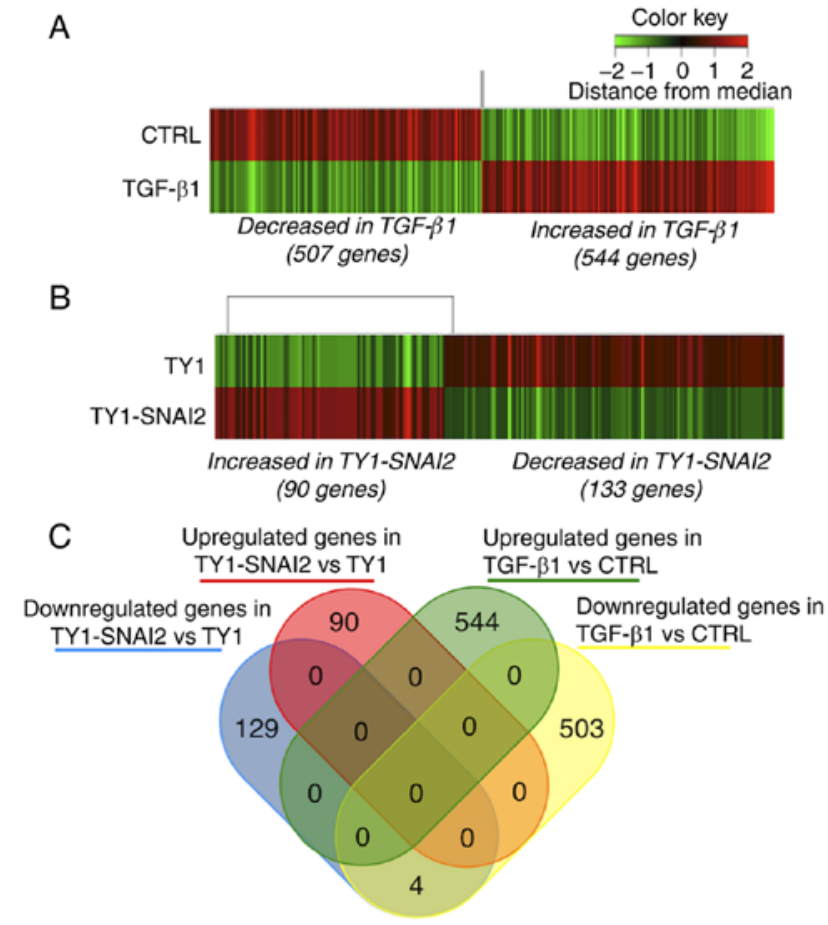

Figure 4. Gene expression changes in HaCaT cells treated with TGF- $\beta 1$ or in those in which expression of SNAI2 was induced. (A) Heatmap of differentially expressed genes in TGF- $\beta 1$ treated HaCaT cells compared with control cells. Red represents high relative expression and green represents low expression. (B) Heatmap of differentially expressed genes in the TY1-SNAI2 expressing cells compared with control cells (TY1-tag expressing cells). Red represents high relative expression, and green represents low expression. (C) Venn diagram showing the number of upregulated and downregulated genes in TGF- $\beta 1$ treated HaCaT cells and TY1-SNAI2-expressing cells. TGF- $\beta 1$, transforming growth factor- $\beta 1$; SNAI2, Slug; CTRL, control (non-treated); TY-1, TY1-tag (TY1-tag expressing).

commonly downregulated genes and no other overlap was found. These data suggested that SNAI2 was not involved in the transcription program of TGF- $\beta 1$-induced EMT in HaCaT cells.

GO and KEGG pathway enrichment analysis of differentially expressed genes. GO and KEGG pathway enrichment analyses were performed to identify the biological attributes of differentially expressed genes in TGF- $\beta 1$-treated $\mathrm{HaCaT}$ cells and SNAI2-overexpressing HaCaT cells. The top 5 enriched terms for the 544 genes upregulated by TGF- $\beta 1$ treatment are shown in Fig. 5A. These genes were found to be significantly enriched in biological processes, such as 'cell adhesion' (GO:0007155), 'tissue morphogenesis' (GO:0048729) and 'extracellular matrix (ECM) organization' (GO:0030198), as well as in cellular components, including 'ECM' (GO:0031012), 'collagen-containing ECM' (GO:0062023), 'adherens junction' (GO:0005912), 'anchoring junction' (GO:0070161) and 'cell-substrate junction' (GO:0030055). In terms of molecular function, the upregulated genes were enriched in 'ECM structural constituent' (GO:0005201), 'integrin binding' (GO:0005102), 'signaling receptor binding' (GO:0005102), 'structural molecule activity' (GO:0005198) and 'fibronectin binding' (GO:0001968). KEGG pathway analysis revealed a strong association of the upregulated genes with pathways, such as focal adhesion (BSID:83067), ECM-receptor interaction (BSID:83068) and the regulation of actin cytoskeleton (BSID:83089) (Fig. 5A). The top 5 downregulated GO terms and KEGG pathway results are illustrated in Fig. 5B. The downregulated genes were significantly enriched in biological processes, such as the 'cell cycle' (GO:0007049), 'nuclear division' (GO:0000280) and 'mitosis' (GO:0000278), in cellular components such as the 'chromosome region' (GO:0098687), and in molecular functions, including several enzymatic activities associated with DNA. Similarly, the KEGG pathway analysis revealed significant enrichment of downregulated genes in the cell cycle (BSID:83054). Although the GO and KEGG pathway enrichment analyses were performed for 90 SNAI2-upregulated genes and 133 SNAI2-downregulated genes, no significant GO terms or pathways were found in either the upregulated or downregulated genes (data not shown). These results suggested that TGF- $\beta 1$ induced dynamic transcriptomic alterations, which led to the EMT features in HaCaT cells.

Identification of transcription factors regulating the differentially expressed genes. Finally, a transcription factor enrichment analysis (ChEA3) was conducted to determine which transcription factors are likely to regulate differentially expressed genes in TGF- $\beta 1$-treated $\mathrm{HaCaT}$ cells. The top 5 transcription factors responsible for the expression of the TGF- $\beta 1$-upregulated genes were identified as transcription factor 12 (TCF12), nuclear factor I C (NFIC), GATA binding protein 3 (GATA3), FOS like 2 (FOSL2), AP-1 transcription factor subunit and TEA domain transcription factor 4 (TEAD4; Fig. 6A). In addition, E2F transcription factor 4 (E2F4), forkhead box M1 (FOXM1), interferon regulatory factor 3 (IRF3), nuclear transcription factor $\mathrm{Y}$ subunit $\beta$ (NFYB) and nuclear transcription factor Y subunit $\alpha$ (NFYA) were predicted to downregulate gene expression in response to TGF- $\beta 1$ treatment (Fig. 6B). These data suggest that multiple transcription factors are involved in the transcription program of TGF- $\beta 1$-induced EMT in HaCaT cells.

\section{Discussion}

The EMT-associated transcription program is known to be triggered by the induction of EMT transcription factors (5). The present study demonstrated that the levels of SNAI2 protein, but not other EMT transcription factors were increased during EMT induced by TGF- $\beta 1$ treatment in HaCaT cells. SNAI2 has been known to stimulate keratinocyte motility in response to the release of epidermal growth factor signaling, as well as maintain the undifferentiated status of basal cells in squamous epithelial tissues $(6-8,22)$. In addition, SNAI2 dysregulation has been linked to the progression of oral tongue cancers and EMT in the oral squamous cell carcinoma cells, such as SCC9 and UM1 cells (23). Hence, it was speculated that SNAI2 plays an important role in controlling the EMT-related gene expression in $\mathrm{HaCaT}$ cells. In the present study, SNAI2-overexpressing and -knockdown HaCaT cells were generated to investigate how the phenotypic characteristics of $\mathrm{HaCaT}$ cells were affected by SNAI2. The findings of this study revealed that unlike TGF- $\beta 1$ signaling, overexpressed SNAI2 is not able to induce EMT features and is not needed to promote the EMT program induced by TGF- $\beta 1$ in $\mathrm{HaCaT}$ 
A
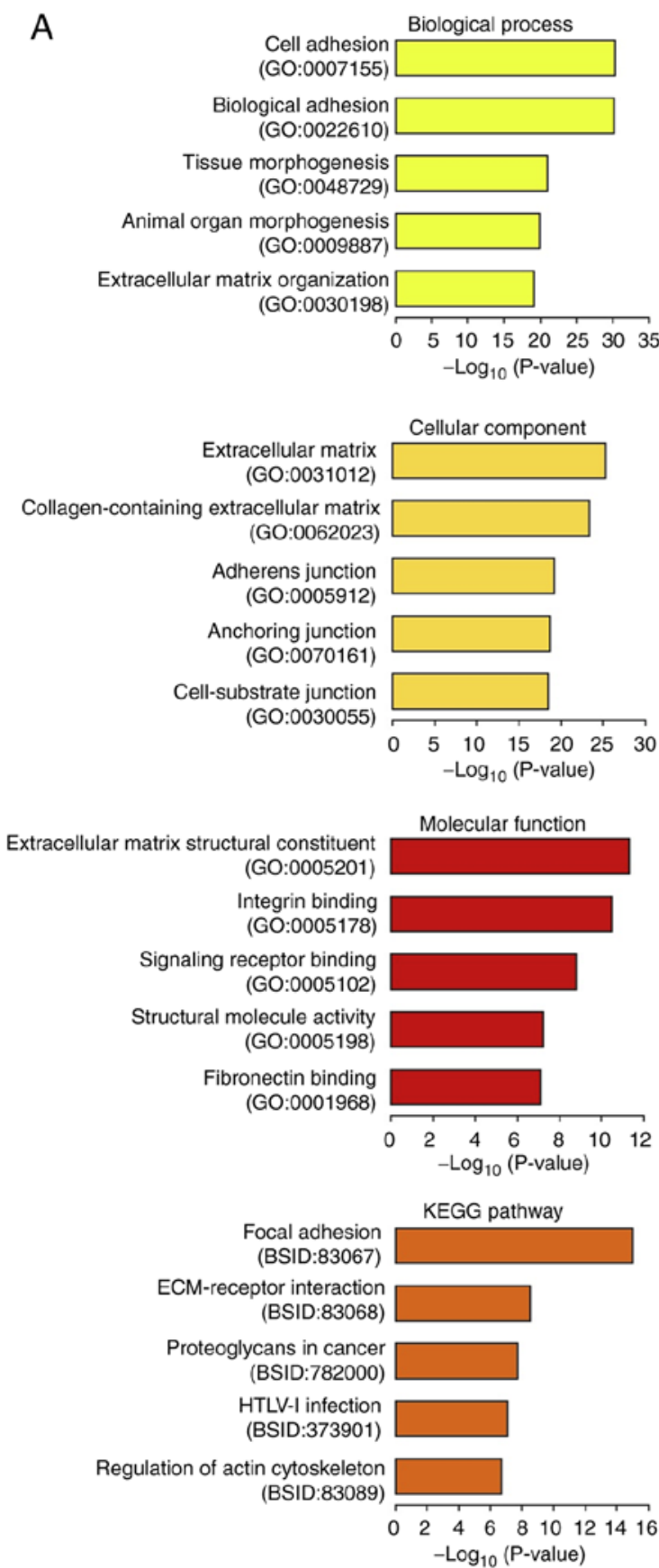

B
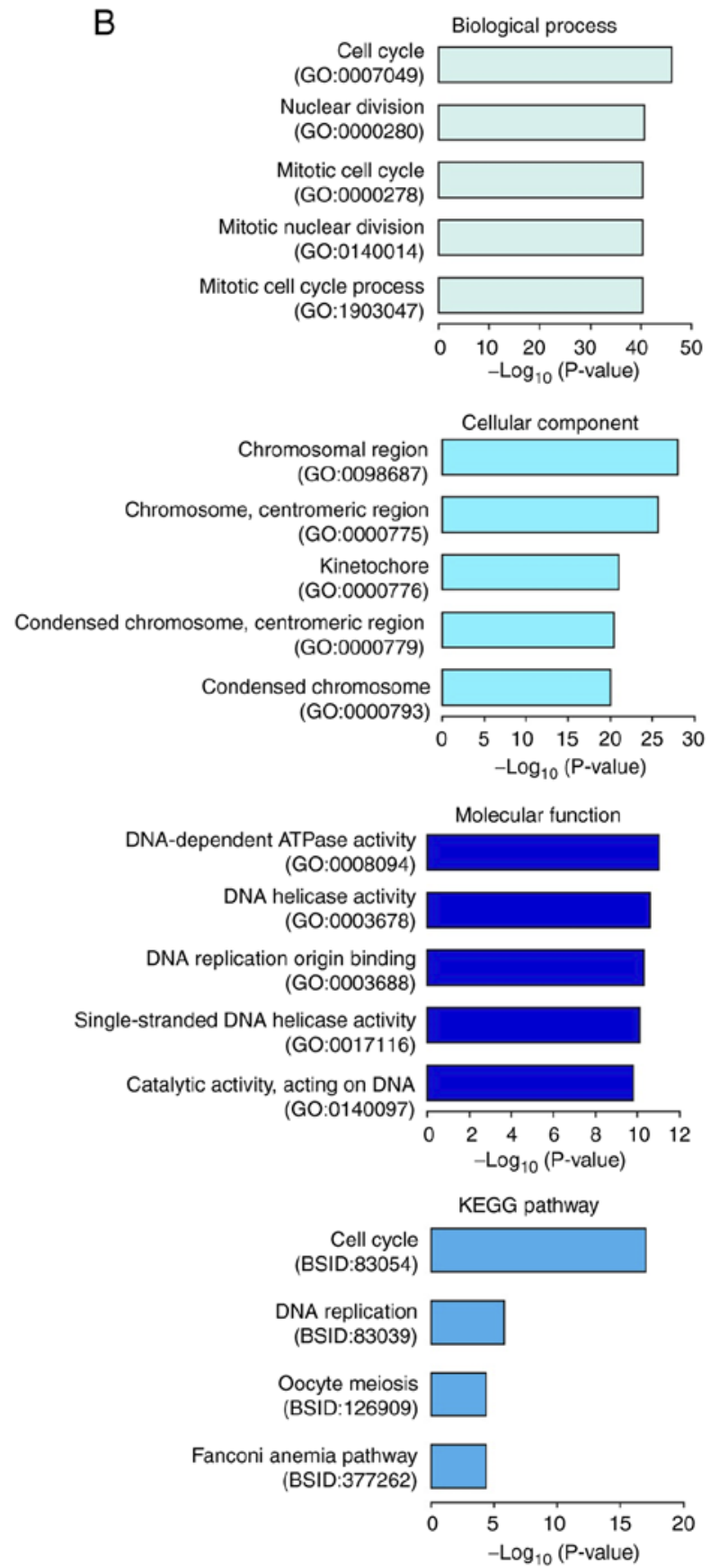

Figure 5. Enrichment analysis of differentially expressed genes in HaCaT cells treated with TGF- $\beta 1$. Functional enrichment analysis of (A) 544 upregulated genes and (B) 507 downregulated genes in TGF- $\beta 1$ treated HaCaT cells. Top 5 enriched GO terms associated with biological process, cellular components, KEGG pathways and molecular functions. Bars represent the negative $\log _{10}$ of the P-value. TGF- $\beta 1$, transforming growth factor- $\beta 1$; GO, Gene Ontology; KEGG, Kyoto Encyclopedia of Genes and Genomes.

cells. However, the results demonstrated that the epithelial markers claudin 1 and keratin 13 as well as the transcription factor SNAI2 were reciprocally expressed in $\mathrm{HaCaT}$ cells. It has been reported that keratin 13 is epigenetically silenced by TGF- $\beta 1$ in HaCaT cells (10); that study raised the possibility that SNAI2 may serve a role in the regulatory mechanism of keratin 13 expression. Claudin 1 is a major component of tight junction complexes in epithelial cells (20). Claudin 1 mRNA levels were not changed by either SNAI2 overexpression or knockdown (data not shown), it is possible that SNAI2 negatively regulates claudin 1 by affecting its translation rate or protein stability. It is also likely that SNAI2 plays different roles in a context-dependent manner and participates in the modulation of epithelial traits, but not the EMT program in non-transformed $\mathrm{HaCaT}$ cells.

This study investigated differentially expressed genes in TGF- $\beta 1$-treated HaCaT cells and SNAI2-overexpressing HaCaT cells. Functional GO annotations and KEGG pathway analysis of genes upregulated by TGF- $\beta 1$-treatment clearly demonstrated enrichment of gene sets associated with cell-matrix adhesions. The genes downregulated by TGF- $\beta 1$-treatment in this study were involved in the cell cycle. These transcriptomic changes induced by TGF- $\beta 1$-treatment should be translated into phenotypic 

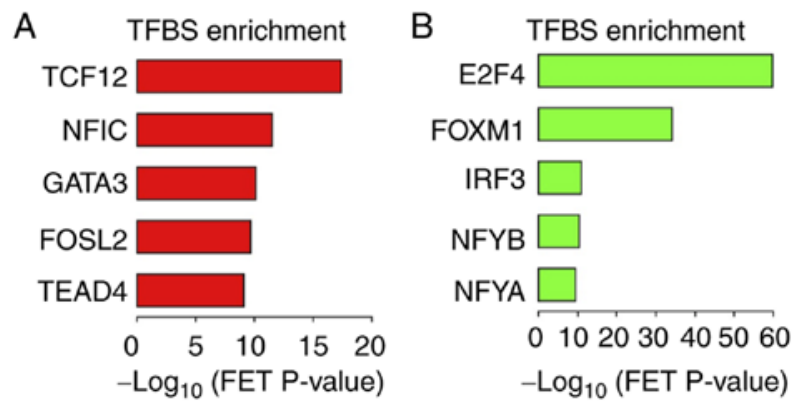

Figure 6. Transcription factor binding site enrichment analysis of differentially expressed genes in $\mathrm{HaCaT}$ cells treated with TGF- $\beta 1$. Top 5 transcription factors associated with (A) 544 upregulated genes and (B) 507 downregulated genes in TGF- $\beta 1$ treated HaCaT cells. Bars represent the negative $\log _{10}$ of the FET P-value. TGF- $\beta 1$, transforming growth factor- $\beta 1$; TFBS, transcription factor binding site; FET, Fisher's exact test; TCF12, transcription factor 12 NFIC, nuclear factor I C; GATA 3, GATA binding protein 3; FOSL2, FOS like 2, AP-1 transcription factor subunit; TEAD 4, TEA domain transcription factor 4; E2F4, E2F transcription factor 4; FOXM1, forkhead box M1 IRF3, interferon regulatory factor 3; NFYB, nuclear transcription factor $\mathrm{Y}$ subunit $\beta$; NFYA, nuclear transcription factor Y subunit $\alpha$.

alterations during EMT in $\mathrm{HaCaT}$ cells. However, no significant GO terms and pathway were found in the differentially expressed genes of SNAI2-overexpressing cells, which did not show EMT-like phenotypes. In addition, there was almost no overlap of differentially expressed genes between the TGF- $\beta 1$-treated cells and the SNAI2-overexpressing cells. These findings suggested that SNAI2 is not involved in the transcriptional regulation of the TGF- $\beta 1$-induced EMT program in $\mathrm{HaCaT}$ cells. A previous study reported that ETS proto-oncogene 2 transcription factor, hepatocyte nuclear factor- $4 \alpha$, and JunB proto-oncogene AP-1 transcription factor subunit, but not canonical EMT transcription factors such as SNAI1/2, TWIST1/2, and ZEB1/2, cooperatively control TGF- $\beta 1$-induced EMT in human lung cancer A549 cells (24). Hence, in the present study, ChEA3 analysis using the lists of differentially expressed genes was performed to identify candidates for master transcription factors that regulate TGF- $\beta 1$-induced EMT in HaCaT cells. As expected, canonical EMT transcription factors were not listed as master transcription factors responsible for the differentially expressed genes. TCF12, NFIC, GATA3, FOSL2, and TEAD4 were identified as the major regulators of the upregulated genes. TCF12 is a member of basic helix-loop-helix (HLH) protein family and mutations in TCF12 have been linked to coronal craniosynostosis (25). NFIC, which belongs to the CTF/NF-I family, has been reported to transcriptionally regulate the $\alpha 6$ integrin gene during corneal wound healing (26). GATA3 and FOSL2 have been reported to regulate the differentiation process of keratinocytes $(27,28)$. TEAD4 is a transcription factor of Hippo pathway signaling involved in the control of organ size and its overexpression has been associated with tumor progression of head and neck squamous cell carcinomas and EMT in cancer cell lines, such as Cal27 and Fadu cells (29). In addition, E2F4, FOXM1, IRF3, NFY and NFYA were identified as the major regulators of the downregulated genes. E2F4 and FOXM1 have been reported to regulate cell cycle genes, including MYC proto-oncogene bHLH transcription factor, cyclin B1, cyclin dependent kinase inhibitor 1A, and to form complexes with the effector SMAD proteins ( $\operatorname{Smad} 3 / \mathrm{Smad} 4)$ to modulate the cellular response to TGF- $\beta 1$ (30-33). IRF3 is a transcription factor that serves an important role in the innate immune response of cells, such as keratinocytes (34). NFYA and NFYB form complexes with NFYC and bind to the CCAAT box motif of the target genes (35). NFYs have been found to regulate the promoter activity of $\mathrm{p} 63$, which is a master regulator of epidermal development (36). Although the results of the present study suggest that the identified transcription factors, but not the canonical EMT transcription factors, control the TGF- $\beta 1$-induced transcription program of $\mathrm{HaCaT}$ cells in a cooperative manner, the functional roles of these factors need to be experimentally validated. Moreover, it is possible that additional undiscovered factors could be involved in the regulation of gene expression and chromatin structure.

In conclusion, the present study demonstrated that SNAI2 is not essential for TGF- $\beta 1$-induced EMT in HaCaT cells and that multiple transcription factors, but not canonical EMT transcription factors, may cooperatively regulate the EMT-related gene expression induced by TGF- $\beta 1$. Although further studies are required to clarify the understanding of molecular events in EMT, the findings of the present study provide insights into the molecular mechanisms underlying the EMT transcription program, and the therapeutic target of EMT in diseases and pathological conditions.

\section{Acknowledgements}

We thank Dr Kazuya Yamagata (Department of Medical Biochemistry, Faculty of Life Sciences, Kumamoto University, Kumamoto, Japan) for providing the pSIREN-RetroQ vector, Dr Tetsuro Ikebe (Department of Oral and Maxillofacial Surgery,Fukuoka Dental College,Fukuoka, Japan) for providing the HaCaT cells and Dr Tsuyako Ohkubo (Educational Support and Institutional Research Office, Fukuoka Nursing College, Fukuoka, Japan) for providing the NIH3T3 cells.

\section{Funding}

This study was supported by JSPS KAKENHI (grant no. JP17K11659).

\section{Availability of data and materials}

The datasets used and/or analyzed during the current study are available from the corresponding author on reasonable request.

\section{Authors' contributions}

YM and MH conceived and designed the study. YM, YN, YT and $\mathrm{MH}$ performed the experiments. YM, YN, YT, KO, ST and $\mathrm{MH}$ analyzed the data. $\mathrm{YM}$ and $\mathrm{MH}$ wrote the manuscript. YM and MH confirm the authenticity of all the raw data All authors read and approved the final manuscript.

\section{Ethics approval and consent to participate}

Not applicable. 


\section{Patient consent for publication}

Not applicable.

\section{Competing interests}

The authors declare that they have no competing interests.

\section{References}

1. Hay ED: An overview of epithelio-mesenchymal transition. Acta Anat (Basel) 154: 8-20, 1995

2. Kalluri R and Weinberg RA: The basics of epithelial-mesenchymal transition. J Clin Invest 119: 1420-1428, 2009.

3. Thiery JP, Acloque H, Huang RY and Nieto MA: Epithelialmesenchymal transitions in development and disease. Cell 139: 871-890, 2009

4. Xu J, Lamouille S and Derynck R: TGF-beta-induced epithelial to mesenchymal transition. Cell Res 19: 156-172, 2009.

5. Lamouille S, Xu J and Derynck R: Molecular mechanisms of epithelial-mesenchymal transition. Nat Rev Mol Cell Biol 15 178-196, 2014

6. Kusewitt DF, Choi C, Newkirk KM, Leroy P, Li Y, Chavez MG and Hudson LG: Slug/Snai2 is a downstream mediator of epidermal growth factor receptor-stimulated reepithelialization. J Invest Dermatol 129: 491-495, 2009.

7. Arnoux V, Nassour M, L'Helgoualc'h A, Hipskind RA and Savagner P: Erk5 controls Slug expression and keratinocyte activation during wound healing. Mol Biol Cell 19: 4738-4749, 2008

8. Hudson LG, Newkirk KM, Chandler HL, Choi C, Fossey SL, Parent AE and Kusewitt DF: Cutaneous wound reepithelialization is compromised in mice lacking functional Slug (Snai2) J Dermatol Sci 56: 19-26, 2009.

9. Shaw TJ and Martin P: Wound repair: A showcase for cell plasticity and migration. Curr Opin Cell Biol 42: 29-37, 2016.

10. Hatta M, Miyake Y, Uchida K and Yamazaki J: Keratin 13 gene is epigenetically suppressed during transforming growth factor- $\beta 1$-induced epithelial-mesenchymal transition in a human keratinocyte cell line. Biochem Biophys Res Commun 496 381-386, 2018

11. Livak KJ and Schmittgen TD: Analysis of relative gene expression data using real-time quantitative PCR and the 2(-Delta Delta C(T)) method. Methods 25: 402-408, 2001

12. Bolstad BM, Irizarry RA, Astrand M and Speed TP: A comparison of normalization methods for high density oligonucleotide array data based on variance and bias. Bioinformatics 19 : 185-193, 2003.

13. Gentleman RC, Carey VJ, Bates DM, Bolstad B, Dettling M, Dudoit S, Ellis B, Gautier L, Ge Y, Gentry J, et al: Bioconductor: Open software development for computational biology and bioinformatics. Genome Biol 5: R80, 2004.

14. Quackenbush J: Microarray data normalization and transformation. Nat Genet (Suppl 32): S496-S501, 2002

15. Saeed AI, Sharov V, White J, Li J, Liang W, Bhagabati N, Braisted J, Klapa M, Currier T, Thiagarajan M, et al: TM4: A free, open-source system for microarray data management and analysis. Biotechniques 34: 374-378, 2003.

16. Chen J, Bardes EE, Aronow BJ and Jegga AG: ToppGene Suite for gene list enrichment analysis and candidate gene prioritization. Nucleic Acids Res 37: W305-W311, 2009.

17. Keenan AB, Torre D, Lachmann A, Leong AK, Wojciechowicz ML, Utti V, Jagodnik KM, Kropiwnicki E, Wang Z and Ma'ayan A: ChEA3: Transcription factor enrichment analysis by orthogonal omics integration. Nucleic Acids Res 47: W212-W224, 2019.

18. Zeisberg $M$ and Neilson EG: Biomarker for epithelial-mesenchymal transitions. J Clin Invest 119: 1429-1437, 2009.
19. Jainchill JL, Aaronson SA and Todaro GJ: Murine sarcoma and leukemia viruses: Assay using clonal lines of contact-inhibited mouse cells. J Virol 4: 549-553, 1969.

20. Krause G, Winkler L, Mueller SL, Haseloff RF, Piontek J and Blasig IE: Structure and function of claudins. Biochim Biophys Acta 1778: 631-645, 2008.

21. Moll R, Divo M and Langbein L: The human keratins: Biology and pathology. Histochem Cell Biol 129: 705-733, 2008

22. Mistry DS, Chen Y, Wang Y, Zhang K and Sen GL: SNAI2 controls the undifferentiated state of human epidermal progenitor cells. Stem Cells 32: 3209-3218, 2014.

23. Wang C, Liu X, Huang H, Ma H, Cai W, Hou J, Huang L, Dai Y, $\mathrm{Yu} \mathrm{T}$ and Zhou X: Deregulation of Snai2 is associated with metastasis and poor prognosis in tongue squamous cell carcinoma. Int J Cancer 130: 2249-2258, 2012

24. Chang H, Liu Y, Xue M, Liu H, Du S, Zhang L and Wang P: Synergistic action of master transcription factors controls epithelial-to-mesenchymal transition. Nucleic Acids Res 44: 2514-2527, 2016.

25. Sharma VP, Fenwick AL, Brockop MS, McGowan SJ, Goos JA, Hoogeboom AJ, Brady AF, Jeelani NO, Lynch SA, Mulliken JB, et al: Mutations of TCF12, encoding a basic helix-loop-helix partner of TWIST1, are a frequent cause of coronal craniosynostosis. Nat Genet 45: 304-307, 2013.

26. Gaudreault M, Vigneault F, Gingras ME, Leclerc S, Carrier P, Germain L and Guérin SL: Transcriptional regulation of the human alpha6 integrin gene by the transcription factor NFI during corneal wound healing. Invest Ophthalmol Vis Sci 49: 3758-3767, 2008

27. Masse I, Barbollat-Boutrand L, Kharbili ME, Berthier-Vergnes O, Aubert D and Lamartine J: GATA3 inhibits proliferation and induces expression of both early and late differentiation markers in keratinocytes of the human epidermis. Arch Dermatol Res 306: 201-208, 2014

28. Wurm S, Zhang J, Guinea-Viniegra J, García F, Muñoz J, Bakiri L, Ezhkova E and Wagner EF: Terminal epidermal differentiation is regulated by the interaction of Fra-2/AP-1 with Ezh2 and ERK1/2. Genes Dev 29: 144-156, 2015.

29. Zhang W, Li J, Wu Y, Ge H, Song Y, Wang D, Yuan H, Jiang H, Wang Y and Cheng J: TEAD4 overexpression promotes epithelial-mesenchymal transition and associates with aggressiveness and adverse prognosis in head neck squamous cell carcinoma. Cancer Cell Int 18: 178, 2018.

30. Chen CR, Kang Y, Siegel PM and Massagué J: E2F4/5 and p107 as Smad cofactors linking the TGFbeta receptor to c-myc repression. Cell 110: 19-32, 2002

31. Yagi K, Furuhashi M, Aoki H, Goto D, Kuwano H, Sugamura K, Miyazono K and Kato M: c-myc is a downstream target of the Smad pathway. J Biol Chem 277: 854-861, 2002.

32. Laoukili J, Kooistra MR, Brás A, Kauw J, Kerkhoven RM, Morrison A, Clevers $\mathrm{H}$ and Medema RH: FoxM1 is required for execution of the mitotic programme and chromosome stability. Nat Cell Biol 7: 126-136, 2005.

33. Xue J, Lin X, Chiu WT, Chen YH, Yu G, Liu M, Feng XH, Sawaya R, Medema RH, Hung MC, et al: Sustained activation of SMAD3/SMAD4 by FOXM1 promotes TGF- $\beta$-dependent cancer metastasis. J Clin Invest 124: 564-579, 2014.

34. Hiscott J: Triggering the innate antiviral response through IRF-3 activation. J Biol Chem 282: 15325-15329, 2007.

35. Ly LL, Yoshida $\mathrm{H}$ and Yamaguchi M: Nuclear transcription factor $\mathrm{Y}$ and its roles in cellular processes related to human disease. Am J Cancer Res 3: 339-346, 2013.

36. Romano RA, Birkaya B and Sinha S: Defining the regulatory elements in the proximal promoter of DeltaNp63 in keratinocytes: Potential roles for Sp1/Sp3, NF-Y, and p63. J Invest Dermatol 126 : 1469-1479, 2006.

This work is licensed under a Creative Commons Attribution-NonCommercial-NoDerivatives 4.0 International (CC BY-NC-ND 4.0) License. 